\title{
Online Strongly Convex Optimization with Unknown Delays
}

\author{
Yuanyu Wan \\ National Key Laboratory for Novel Software Technology \\ Nanjing University, Nanjing 210023, China
}

Wei-Wei Tu

4Paradigm Inc., Beijing 100000, China

\author{
Lijun Zhang \\ National Key Laboratory for Novel Software Technology \\ Nanjing University, Nanjing 210023, China
}

\author{
WANYY@LAMDA.NJU.EDU.CN
}

TUWEIWEI@4PARADIGM.COM

ZHANGLJ@LAMDA.NJU.EDU.CN

\begin{abstract}
We investigate the problem of online convex optimization with unknown delays, in which the feedback of a decision arrives with an arbitrary delay. Previous studies have presented a delayed variant of online gradient descent (OGD), and achieved the regret bound of $O(\sqrt{T+D})$ by only utilizing the convexity condition, where $D$ is the sum of delays over $T$ rounds. In this paper, we further exploit the strong convexity to improve the regret bound. Specifically, we first extend the delayed variant of OGD for strongly convex functions, and establish a better regret bound of $O(d \log T)$, where $d$ is the maximum delay. The essential idea is to let the learning rate decay with the total number of received feedback linearly. Furthermore, we consider the more challenging bandit setting, and obtain similar theoretical guarantees by incorporating the classical multi-point gradient estimator into our extended method. To the best of our knowledge, this is the first work that solves online strongly convex optimization under the general delayed setting.

Keywords: Online Convex Optimization, Strongly Convex, Unknown Delays, Regret, Bandit
\end{abstract}

\section{Introduction}

Online convex optimization (OCO) is a prominent paradigm for sequential decision making, which has been successfully applied to many tasks such as portfolio selection (Blum and Kalai, 1999; Agarwal et al., 2006; Luo et al., 2018) and online advertisement (McMahan et al., 2013; He et al., 2014; Juan et al., 2017). At each round $t$, a player selects a decision $\mathbf{x}_{t}$ from a convex set $\mathcal{X}$. Then, an adversary chooses a convex loss function $f_{t}(\mathbf{x}): \mathcal{X} \mapsto \mathbb{R}$, and incurs a loss $f_{t}\left(\mathbf{x}_{t}\right)$ to the player. The performance of the player is measured by the regret

$$
R_{T}=\sum_{t=1}^{T} f_{t}\left(\mathbf{x}_{t}\right)-\min _{\mathbf{x} \in \mathcal{X}} \sum_{t=1}^{T} f_{t}(\mathbf{x})
$$

which is the gap between the cumulative loss of the player and an optimal fixed decision.

Online gradient descent (OGD) proposed by Zinkevich $(2003)$ is a standard method for minimizing the regret. For convex functions, Zinkevich (2003) showed that OGD attains an $O(\sqrt{T})$ regret bound. If the functions are strongly convex, Hazan et al. (2007) proved that OGD can achieve a better regret bound of $O(\log T)$. The $O(\sqrt{T})$ and $O(\log T)$ bounds have been proved to be minimax optimal for convex and strongly convex functions, respectively 
(Abernethy et al., 2008). However, the standard OCO assumes that the loss function $f_{t}(\mathbf{x})$ is revealed to the player immediately after making the decision $\mathbf{x}_{t}$, which does not account for the possible delay between the decision and feedback in various practical applications. For example, in online advertisement, the decision is about the strategy of serving an ad to a user, and the feedback required to update the decision usually is whether the ad is clicked or not (McMahan et al., 2013). But, after seeing the ad, the user may take some time to give feedback. Moreover, there may not exist a button for the negative feedback, which is not determined unless the user does not click the ad after a sufficiently long period $(\mathrm{He}$ et al. 2014).

To address the above challenge, Quanrud and Khashabi (2015) proposed delayed OGD (DOGD) for OCO with unknown delays, and attained the $O(\sqrt{T+D})$ regret bound, where $D$ is the sum of delays over $T$ rounds. Similar to OGD, in each round $t$, DOGD queries the gradient $\nabla f_{t}\left(\mathbf{x}_{t}\right)$, but according to the delayed setting, it will be received at the end of round $t+d_{t}-1$ where $d_{t} \geq 1$ is an unknown integer. By the same token, gradients queried in previous rounds may be received at the end of round $t$, and DOGD updates the decision $\mathbf{x}_{t}$ with the sum of received gradients. Recently, Li et al. (2019) further considered the more challenging bandit setting, and proposed delayed bandit gradient descent (DBGD) with $O(\sqrt{T+D})$ regret bound. Specifically, DBGD queries each function $f_{t}(\mathbf{x})$ at $n+1$ points where $n$ is the dimensionality, and approximates the gradient by applying the classical $(n+1)$-point gradient estimator (Agarwal et al. 2010) to each received feedback. At the end of round $t$, different from DOGD that only updates the decision $\mathbf{x}_{t}$ once, DBGD repeatedly updates the decision $\mathbf{x}_{t}$ with each approximate gradient. While DOGD and DBGD can handle unknown delays for the full information and bandit settings respectively, it remains unclear whether the strong convexity of loss functions can be utilized to achieve a better regret bound.

We notice that Khashabi et al. (2016) have tried to exploit the strong convexity for DOGD, but failed because they discovered mistakes in their proof. In this paper, we provide an affirmative answer by proposing a variant of DOGD for strongly convex functions, namely DOGD-SC, which achieves a regret bound of $O(d \log T)$, where $d$ is the maximum delay. To this end, we refine the learning rate used in the original DOGD with a new one that decays with the total number of received feedback linearly, which is able to exploit the strong convexity. For a small $d=O(1)$, our $O(d \log T)$ regret bound is significantly better than the $O(\sqrt{T+D})$ regret bound established by only using the convexity condition. Furthermore, to handle the bandit setting, we propose a bandit variant of DOGD-SC by combining with the $(n+1)$-point gradient estimator (Agarwal et al., 2010). In each round, we only update the decision once with the sum of approximate gradients, which could be more efficient than DBGD (Li et al., 2019). Our theoretical analysis reveals that the bandit variant of DOGD-SC can also obtain the $O(d \log T)$ regret bound for strongly convex functions, which is better than the $O(\sqrt{T+D})$ regret bound of DBGD.

\section{Related Work}

In this section, we briefly review the related work about OCO with delayed feedback, in which the feedback for the decision $\mathbf{x}_{t}$ is received at the end of round $t+d_{t}-1$. 


\subsection{The Standard OCO}

If $d_{t}=1$ for all $t \in[T]$, OCO with delayed feedback is reduced to the standard OCO, in which various algorithms have been proposed to minimize the regret under the full information and bandit settings (Shalev-Shwartz, 2011, Hazan, 2016). In the full information setting, by using the gradient of each function, the standard OGD achieves $O(\sqrt{T})$ and $O(\log T)$ regret bounds for convex (Zinkevich, 2003) and strongly convex functions (Hazan et al., 2007), respectively. For the bandit setting, where only the function value is available to the player, Agarwal et al. (2010) proposed to approximate the gradient by querying the function at two points or $n+1$ points. Moreover, they showed that OGD with the approximate gradient can also attain $O(\sqrt{T})$ and $O(\log T)$ regret bounds for convex and strongly convex functions, respectively.

\subsection{OCO with Fixed and Known Delays}

To handle the case that each feedback arrives with a fixed and known delay $d$, i.e., $d_{t}=d$ for all $t \in[T]$, Weinberger and Ordentlich $(2002)$ divide the total $T$ rounds into $d$ subsets $\mathcal{T}_{1}, \cdots, \mathcal{T}_{d}$, where $\mathcal{T}_{i}=\{i, i+d, i+2 d, \cdots\} \cap[T]$ for $i=1, \cdots, d$. Over rounds in the subset $\mathcal{T}_{i}$, they maintain an instance $\mathcal{A}_{i}$ of a base algorithm $\mathcal{A}$. If the base algorithm $\mathcal{A}$ enjoys a regret bound of $R_{\mathcal{A}}(T)$ for the standard OCO, Weinberger and Ordentlich (2002) showed that their method attains a regret bound of $d R_{\mathcal{A}}(T / d)$. By setting the base algorithm $\mathcal{A}$ as OGD, the regret bounds could be $O(\sqrt{d T})$ for convex functions and $O(d \log T)$ for strongly convex functions, respectively. However, since this method needs to maintain $d$ instances in total, the space complexity is $d$ times as much as that of the base algorithm.

By contrast, Langford et al. (2009) proposed a more efficient method by simply performing the gradient descent step with a delayed gradient, and also achieved the $O(\sqrt{d T})$ and $O(d \log T)$ regret bounds for convex and strongly convex functions, respectively. Moreover, Shamir and Szlak (2017) combined the fixed delay with the local permutation setting, in which the order of the functions can be modified by a distance of at most $M$. When $M \geq d$, they improved the regret bound to $O\left(\sqrt{T}\left(1+\sqrt{d^{2} / M}\right)\right)$ for convex functions.

\subsection{OCO with Arbitrary but Time-stamped Delays}

Several previous studies considered another delayed setting, in which each feedback could be delayed by arbitrary rounds, but is time-stamped when it is received. Specifically, Mesterharm (2005) focused on the online classification problem, and analyzed the bound for the number of mistakes. Joulani et al. (2013) further proposed to solve OCO under this delayed setting by extending the method of Weinberger and Ordentlich (2002). However, similar to Weinberger and Ordentlich (2002), the method proposed by Joulani et al. (2013) needs to maintain multiple instances of a base algorithm, which could be prohibitively resource-intensive. Recently, if each delay $d_{t}$ grows as $o\left(t^{c}\right)$ for some known $c<1$, Héliou et al. (2020) employed the one-point gradient estimator (Flaxman et al. 2005$)$ to propose a new method for the bandit setting, and established an expected regret bound of $\tilde{O}\left(T^{3 / 4}+T^{2 / 3+c / 3}\right)$ for convex functions. 


\subsection{OCO with Unknown Delays}

Furthermore, Quanrud and Khashabi (2015) considered a more general delayed setting, in which each feedback could be delayed arbitrarily and the time stamp of each feedback could also be unknown, and proposed an efficient method called DOGD. The main idea of DOGD is to query the gradient $\nabla f_{t}\left(\mathbf{x}_{t}\right)$ at each round $t$, and update the decision $\mathbf{x}_{t}$ with the sum of those gradients queried at the set of rounds $\mathcal{F}_{t}=\left\{k \mid k+d_{k}-1=t\right\}$. Different from Joulani et al. (2013), DOGD enjoys the $O(\sqrt{T+D})$ regret bound without any assumption about delays, where $D$ is the sum of delays over $T$ rounds. Khashabi et al. (2016) tried to improve the regret bound of DOGD for strongly convex functions, but did not provide a rigorous analysis. Recently, Li et al. (2019) proposed DBGD to handle the more challenging bandit setting. In each round $t, \mathrm{DBGD}$ queries the function $f_{t}(\mathbf{x})$ at $n+1$ points, and repeatedly updates the decision $\mathbf{x}_{t}$ with each approximate gradient computed by applying the $(n+1)$-point gradient estimator (Agarwal et al., 2010) to each feedback received from the set of rounds $\mathcal{F}_{t}=\left\{k \mid k+d_{k}-1=t\right\}$. This method also attains a regret bound of $O(\sqrt{T+D})$, but needs to update the decision $\left|\mathcal{F}_{t}\right|$ times in each round $t$.

If the feedback of each decision $\mathbf{x}_{t}$ is the entire loss function $f_{t}(\mathbf{x})$, Joulani et al. $(2016)$ provided an algorithmic framework for extending a base algorithm to the delayed setting. By combining the proposed framework with adaptive online algorithms (McMahan and Streeter, 2010 Duchi et al., 2011), they improved the $O(\sqrt{T+D})$ regret bound to a data-dependent one. If the decision set is unbounded and the order of the received feedback keeps the same as the case without delay, an adaptive algorithm and the data-dependent regret bound for the delayed setting were already presented by McMahan and Streeter $(2014)$. In the worst case, these data-dependent regret bounds would reduce to $O(\sqrt{T+D})$ or $O(\sqrt{d T})$ where $d$ is the maximum delay, which cannot benefit from the strong convexity.

Although there are many studies about OCO with unknown delays, it remains unclear whether the strong convexity can be utilized to improve the regret bound. This paper provides an affirmative answer by establishing the $O(d \log T)$ regret bound for strongly convex functions.

\section{Main Results}

In this section, we first present DOGD-SC, a variant of DOGD for strongly convex functions, which improves the regret bound. Then, we extend DOGD-SC to the bandit setting.

\subsection{DOGD-SC with Improved Regret}

Following previous studies (Shalev-Shwartz, 2011; Hazan, 2016), we introduce some common assumptions.

Assumption 1 Each loss function $f_{t}(\mathbf{x})$ is L-Lipschitz over $\mathcal{X}$, i.e., $\left|f_{t}(\mathbf{x})-f_{t}(\mathbf{y})\right| \leq$ $L\|\mathbf{x}-\mathbf{y}\|$, for any $\mathbf{x}, \mathbf{y} \in \mathcal{X}$, where $\|\cdot\|$ denotes the Euclidean norm.

Assumption 2 The radius of the convex decision set $\mathcal{X}$ is bounded by $R$, i.e., $\|\mathbf{x}\| \leq R$, for any $\mathbf{x} \in \mathcal{X}$. 


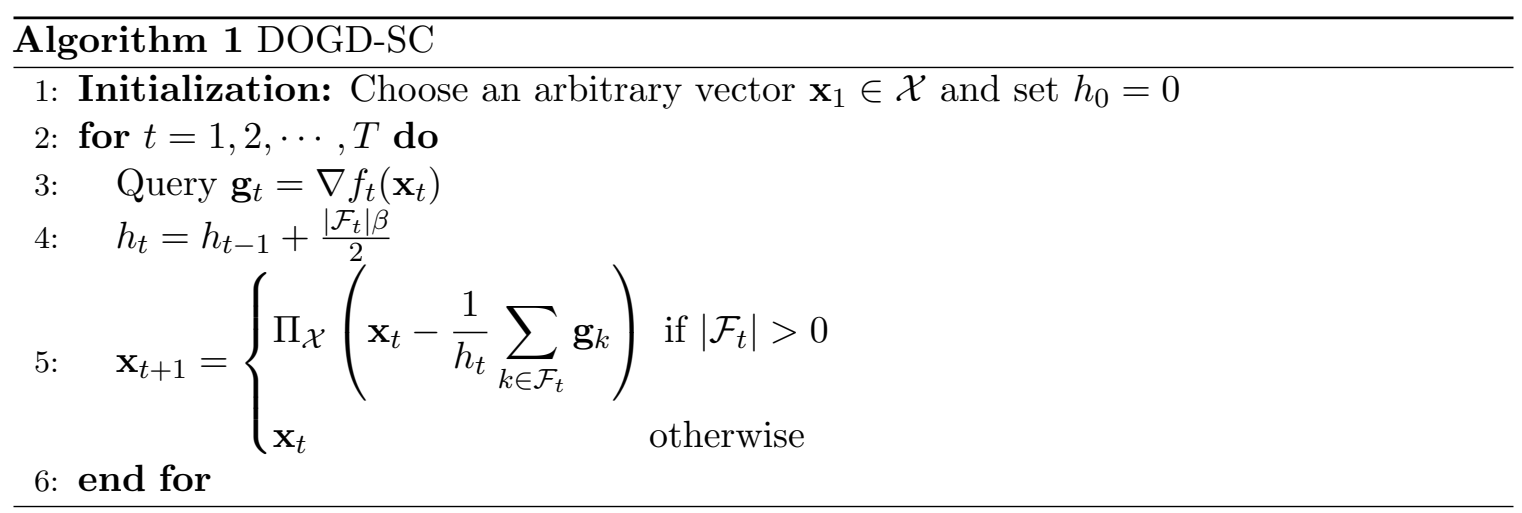

Assumption 3 Each loss function $f_{t}(\mathbf{x})$ is $\beta$-strongly convex over $\mathcal{X}$, i.e., for any $\mathbf{x}, \mathbf{y} \in \mathcal{X}$

$$
f_{t}(\mathbf{y}) \geq f_{t}(\mathbf{x})+\nabla f_{t}(\mathbf{x})^{\top}(\mathbf{y}-\mathbf{x})+\frac{\beta}{2}\|\mathbf{x}-\mathbf{y}\|^{2} .
$$

To handle OCO with unknown delays, DOGD (Quanrud and Khashabi, 2015) first arbitrarily chooses $\mathbf{x}_{1}$ from $\mathcal{X}$. In each round $t$, it queries the gradient $\mathbf{g}_{t}=\nabla f_{t}\left(\mathbf{x}_{t}\right)$, and then receives the gradient queried in the set of rounds $\mathcal{F}_{t}=\left\{k \mid k+d_{k}-1=t\right\}$. If $\left|\mathcal{F}_{t}\right|=0$, DOGD keeps the decision unchanged as $\mathbf{x}_{t+1}=\mathbf{x}_{t}$. Otherwise, it updates the decision with the sum of gradients received at this round as

$$
\mathbf{x}_{t+1}=\Pi_{\mathcal{X}}\left(\mathbf{x}_{t}-\eta_{t} \sum_{k \in \mathcal{F}_{t}} \mathbf{g}_{k}\right)
$$

where $\Pi_{\mathcal{X}}(\mathbf{y})=\operatorname{argmin}_{\mathbf{x} \in \mathcal{X}}\|\mathbf{x}-\mathbf{y}\|$ for any vector $\mathbf{y}$ is the projection operation. According to Quanrud and Khashabi (2015), DOGD attains a regret bound of $O(\sqrt{T+D})$ by using a constant learning rate $\eta_{t}=O(1 / \sqrt{T+D})$ for all $t \in[T]$, where $D$ is the sum of delays and can be estimated on the fly via the standard "doubling trick" (Cesa-Bianchi and Lugosi, 2006).

However, the constant learning rate cannot utilize the strong convexity of the loss functions. In the standard OCO where $\mathcal{F}_{t}=\{t\}$ for any $t \in[T]$, Hazan et al. (2007) have established the $O(\log T)$ regret bound for $\beta$-strongly convex functions by setting $\eta_{t}=1 /(\beta t)$. A significant property of the learning rate is that the inverse of $\eta_{t}$ is increasing by the modulus of the strong convexity of $f_{t}(\mathbf{x})$ per round, i.e.,

$$
\frac{1}{\eta_{t+1}}-\frac{1}{\eta_{t}}=\beta
$$

Inspired by (1), we initialize $\frac{1}{\eta_{0}}=0$ and update it as

$$
\frac{1}{\eta_{t+1}}=\frac{1}{\eta_{t}}+\frac{\left|\mathcal{F}_{t}\right| \beta}{2}
$$

where $\left|\mathcal{F}_{t}\right| \beta$ is the modulus of the strong convexity of $\sum_{k \in \mathcal{F}_{t}} f_{k}(\mathbf{x})$, and the constant $1 / 2$ is essential for our analysis. Let $h_{t}=1 / \eta_{t}$ for $t=0, \cdots, T$. The detailed procedures for 
strongly convex functions are summarized in Algorithm 1, which is named as DOGD for strongly convex functions (DOGD-SC).

Let $d=\max \left\{d_{t} \mid t=1, \cdots, T\right\}$ denote the maximum delay. Since there could exist some gradients that arrive after the round $T$, we also define $\mathcal{F}_{t}=\left\{k \mid k+d_{k}-1=t\right\}$ for any $t=T+1, \cdots, T+d-1$. Then, we establish the following theorem regarding the regret of Algorithm 1 .

Theorem 1 Under Assumptions 1, 2 and 3, Algorithm 1 satisfies

$$
R_{T} \leq\left(4 \beta R L+5 L^{2}\right) \frac{d}{\beta}\left(1+\ln \frac{T}{\left|\mathcal{F}_{s}\right|}\right)
$$

where $s=\min \left\{t|t \in[T+d-1],| \mathcal{F}_{t} \mid>0\right\}$.

From Theorem 1, the regret bound of Algorithm 1 is on the order of $O(d \log T)$, which is better than the $O(\sqrt{T+D})$ regret bound established by Quanrud and Khashabi $(2015)$ as long as $d<\sqrt{T+D} / \log T$. Moreover, if $d=O(1)$, our $O(d \log T)$ regret bound is on the same order as the $O(\log T)$ bound for OCO without delay. We note that Khashabi et al. (2016) have tried to use the strong convexity by setting $\eta_{t}=\frac{2}{\beta t\left|\mathcal{F}_{t}\right|}$. However, in this way, there could exist some rounds such that $(t+1)\left|\mathcal{F}_{t+1}\right| \leq t\left|\mathcal{F}_{t}\right|$ and

$$
\frac{1}{\eta_{t+1}}-\frac{1}{\eta_{t}}=\frac{\beta}{2}\left((t+1)\left|\mathcal{F}_{t+1}\right|-t\left|\mathcal{F}_{t}\right|\right) \leq 0
$$

which makes the proof of their Theorem 3.1 problematic.

\subsection{Algorithm for Bandit Setting}

To handle the bandit setting, following previous studies (Agarwal et al., 2010; Saha and Tewari, 2011), we further introduce two assumptions, as follows.

Assumption 4 Let $\mathcal{B}^{n}$ denote the unit Euclidean ball centered at the origin in $\mathbb{R}^{n}$. There exists a constant $r$ such that $r \mathcal{B}^{n} \subseteq \mathcal{X}$.

Assumption 5 Each loss function $f_{t}(\mathbf{x})$ is $\alpha$-smooth over $\mathcal{X}$, i.e., for any $\mathbf{x}, \mathbf{y} \in \mathcal{X}$

$$
f_{t}(\mathbf{y}) \leq f_{t}(\mathbf{x})+\nabla f_{t}(\mathbf{x})^{\top}(\mathbf{y}-\mathbf{x})+\frac{\alpha}{2}\|\mathbf{y}-\mathbf{x}\|^{2} .
$$

In the bandit setting, since only the function value is available to the player instead of the gradient, the problem becomes more challenging. Fortunately, Agarwal et al. (2010) have proposed to approximate the gradient by querying the function at two points or $n+1$ points. To avoid the cost of querying the function many times, one may prefer to adopt the two-point gradient estimator. However, as discussed by Li et al. (2019), the two-point gradient estimator would fail in the general delayed setting, because it requires the time stamp of each feedback, which could be unknown.

As a result, we will utilize the $(n+1)$-point gradient estimator in the general delayed setting. Define

$$
\mathcal{X}_{\delta}=(1-\delta / r) \mathcal{X}=\{(1-\delta / r) \mathbf{x} \mid \mathbf{x} \in \mathcal{X}\}
$$




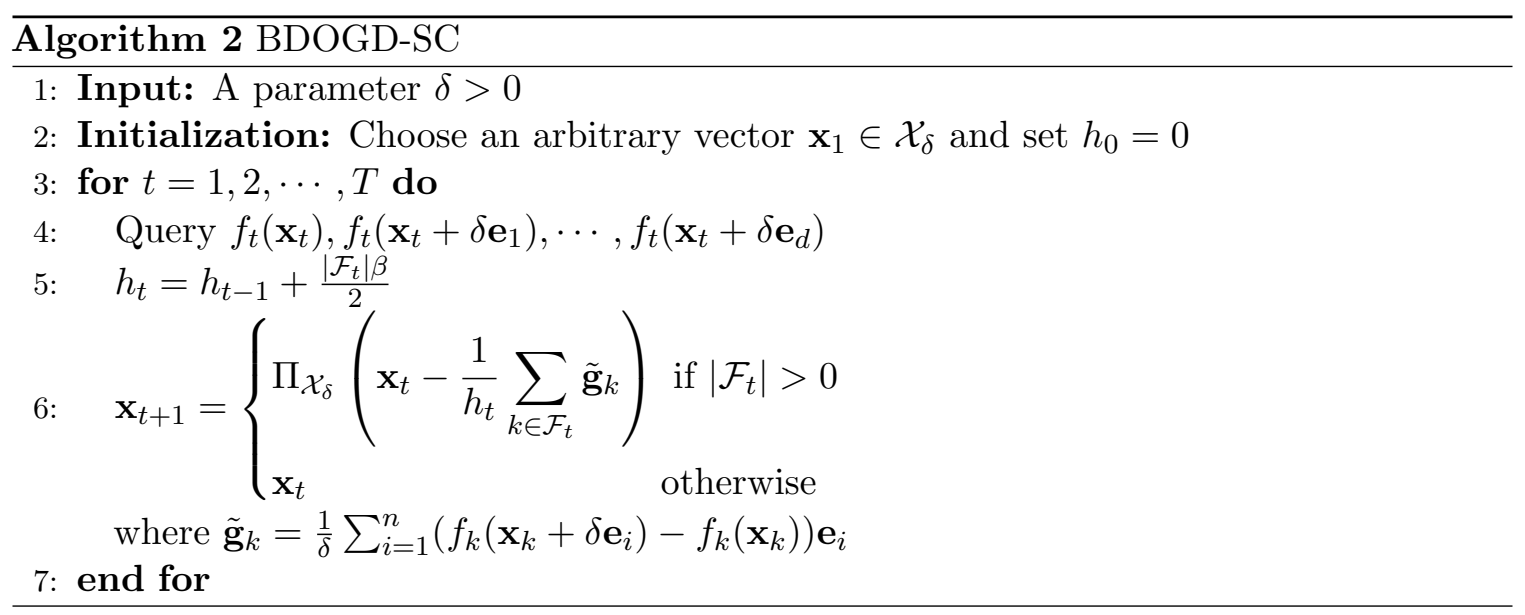

for some $0<\delta<r$. For a function $f(\mathbf{x}): \mathcal{X} \mapsto \mathbb{R}$ and a point $\mathbf{x} \in \mathcal{X}_{\delta}$, the $(n+1)$-point gradient estimator queries

$$
f(\mathbf{x}), f\left(\mathbf{x}+\delta \mathbf{e}_{1}\right), \cdots, f\left(\mathbf{x}+\delta \mathbf{e}_{n}\right)
$$

where $\mathbf{e}_{i}$ denotes the unit vector with the $i$-th entry equal 1 , and estimates the gradient $\nabla f(\mathbf{x})$ by

$$
\tilde{\mathrm{g}}=\frac{1}{\delta} \sum_{i=1}^{n}\left(f\left(\mathbf{x}+\delta \mathbf{e}_{i}\right)-f(\mathbf{x})\right) \mathbf{e}_{i} .
$$

Previous studies have proved that the approximate gradient enjoys the following properties.

Lemma 1 (Lemma 4 in Li et al. (2019)) If $f(\mathbf{x}): \mathcal{X} \mapsto \mathbb{R}$ is L-Lipschitz and $\alpha$-smooth, for any $\mathbf{x} \in \mathcal{X}_{\delta}$, it holds that

$$
\|\tilde{\mathbf{g}}\| \leq \sqrt{n} L \text { and }\|\tilde{\mathbf{g}}-\nabla f(\mathbf{x})\| \leq \frac{\sqrt{n} \alpha \delta}{2}
$$

where $\tilde{\mathbf{g}}$ is computed as (2).

From Lemma 1, the $(n+1)$-point gradient estimator can closely approximate the gradient with a small $\delta$.

To combine Algorithm 1 with the $(n+1)$-point gradient estimator, we need to make three changes as follows. First, at each round $t$, the player queries the function $f_{t}(\mathbf{x})$ at $n+1$ points $\mathbf{x}_{t}, \mathbf{x}_{t}+\delta \mathbf{e}_{1}, \cdots, \mathbf{x}_{t}+\delta \mathbf{e}_{n}$, instead of querying the gradient $\nabla f_{t}\left(\mathbf{x}_{t}\right)$. In this way, the feedback arrives at the end of round $t$ is

$$
\left\{\left\{f_{k}\left(\mathbf{x}_{k}+\delta \mathbf{e}_{i}\right)\right\}_{i=0}^{n} \mid k+d_{k}-1=t\right\}
$$

where $\mathbf{e}_{0}$ is defined as the zero vector. According to (2), we can approximate the gradient $\nabla f_{k}\left(\mathbf{x}_{k}\right)$ as

$$
\tilde{\mathbf{g}}_{k}=\frac{1}{\delta} \sum_{i=1}^{n}\left(f_{k}\left(\mathbf{x}_{k}+\delta \mathbf{e}_{i}\right)-f_{k}\left(\mathbf{x}_{k}\right)\right) \mathbf{e}_{i}
$$


for $k \in \mathcal{F}_{t}$. Therefore, the second change is to update $\mathbf{x}_{t}$ with the sum of gradients estimated from the feedback. Moreover, to ensure that $\mathbf{x}_{t}+\delta \mathbf{e}_{1}, \cdots, \mathbf{x}_{t}+\delta \mathbf{e}_{n} \in \mathcal{X}$, the third change is to limit $\mathbf{x}_{t}$ in the set $\mathcal{X}_{\delta}$ for all $t \in[T]$. Combining the second and third changes, we update the decision as

$$
\mathbf{x}_{t+1}= \begin{cases}\Pi_{\mathcal{X}_{\delta}}\left(\mathbf{x}_{t}-\frac{1}{h_{t}} \sum_{k \in \mathcal{F}_{t}} \tilde{\mathbf{g}}_{k}\right) & \text { if }\left|\mathcal{F}_{t}\right|>0 \\ \mathbf{x}_{t} & \text { otherwise. }\end{cases}
$$

Note that computing $\tilde{\mathbf{g}}_{k}$ and $\sum_{k \in \mathcal{F}_{t}} \tilde{\mathbf{g}}_{k}$ does not require the time stamp of each feedback. The detailed procedures for the bandit setting are summarized in Algorithm 2, which is named as a bandit variant of DOGD-SC (BDOGD-SC).

Since there are $n+1$ decisions selected in each round, following Agarwal et al. (2010), the regret is redefined as the average regret

$$
\tilde{R}_{T}=\frac{1}{n+1} \sum_{t=1}^{T} \sum_{i=0}^{n} f_{t}\left(\mathbf{x}_{t}+\delta \mathbf{e}_{i}\right)-\min _{\mathbf{x} \in \mathcal{X}} \sum_{t=1}^{T} f_{t}(\mathbf{x}) .
$$

We establish the following theorem regarding the average regret of Algorithm 2 .

Theorem 2 Let $\tilde{L}=L+\frac{\sqrt{n} \alpha \delta}{2}$ and $\delta=\frac{c \ln T}{T}$, where $c>0$ is a constant such that $\delta<r$. Under Assumptions 1, 2, 3, 4 and 5, Algorithm 2] ensures

$$
\begin{aligned}
\tilde{R}_{T} \leq & \left(4 \beta R \tilde{L}+5 \tilde{L}^{2}\right) \frac{d}{\beta}\left(1+\ln \frac{T}{\left|\mathcal{F}_{s}\right|}\right)+\sqrt{n} c \alpha R \ln T \\
& +\frac{c L R \ln T}{r}+c L \ln T
\end{aligned}
$$

where $s=\min \left\{t|t \in[T+d-1],| \mathcal{F}_{t} \mid>0\right\}$.

According to Theorem 2, the regret bound of our Algorithm 2 is also on the order of $O(d \log T)$, which is better than the $O(\sqrt{T+D})$ regret bound of DBGD (Li et al., 2019) as long as $d<\sqrt{T+D} / \log T$. Furthermore, in each round $t$, DBGD updates $\left|F_{t}\right|$ times to obtain $\mathbf{x}_{t+1}$, which is more expensive than our Algorithm 2 .

Besides Algorithm 2, an algorithm based on the two-point gradient estimator is developed in the appendix, which can handle the case where the time stamp of each feedback is known.

\section{Analysis}

In this section, we only provide the proof of Theorem 1 and the omitted proofs can be found in the appendix.

\subsection{Preliminaries}

According to Algorithm 1, there could exist some feedback that arrives after the round $T$ and is not used to update the decision. However, it is useful for the analysis. Therefore, we 
perform a virtual update as

$$
\begin{aligned}
& h_{t}=h_{t-1}+\frac{\left|\mathcal{F}_{t}\right| \beta}{2}, \\
& \mathbf{x}_{t+1}= \begin{cases}\Pi_{\mathcal{X}}\left(\mathbf{x}_{t}-\frac{1}{h_{t}} \sum_{k \in \mathcal{F}_{t}} \mathbf{g}_{k}\right) & \text { if }\left|\mathcal{F}_{t}\right|>0 \\
\mathbf{x}_{t} & \text { otherwise }\end{cases}
\end{aligned}
$$

for $t \in[T+1, T+d-1]$.

Then, for any $t \in[T+d-1]$, we define

$$
\mathbf{x}_{t+1}^{\prime}= \begin{cases}\mathbf{x}_{t}-\frac{1}{h_{t}} \sum_{k \in \mathcal{F}_{t}} \mathbf{g}_{k} & \text { if }\left|\mathcal{F}_{t}\right|>0 \\ \mathbf{x}_{t} & \text { otherwise }\end{cases}
$$

Moreover, we define $t^{\prime}=t+d_{t}-1$ for any $t \in[T]$ and $s=\min \left\{t|t \in[T+d-1],| \mathcal{F}_{t} \mid>0\right\}$.

\subsection{Proof of Theorem 1}

Let $\mathbf{x}^{*}=\operatorname{argmin}_{\mathbf{x} \in \mathcal{X}} \sum_{t=1}^{T} f_{t}(\mathbf{x})$. We have

$$
\begin{aligned}
R_{T} & =\sum_{t=1}^{T} f_{t}\left(\mathbf{x}_{t}\right)-\sum_{t=1}^{T} f_{t}\left(\mathbf{x}^{*}\right) \\
& \leq \sum_{t=1}^{T}\left(\nabla f_{t}\left(\mathbf{x}_{t}\right)^{\top}\left(\mathbf{x}_{t}-\mathbf{x}^{*}\right)-\frac{\beta}{2}\left\|\mathbf{x}_{t}-\mathbf{x}^{*}\right\|^{2}\right) \\
& =\sum_{t=1}^{T}\left(\nabla f_{t}\left(\mathbf{x}_{t}\right)^{\top}\left(\mathbf{x}_{t^{\prime}}-\mathbf{x}^{*}\right)-\frac{\beta}{2}\left\|\mathbf{x}_{t}-\mathbf{x}^{*}\right\|^{2}\right)+\sum_{t=1}^{T} \nabla f_{t}\left(\mathbf{x}_{t}\right)^{\top}\left(\mathbf{x}_{t}-\mathbf{x}_{t^{\prime}}\right) \\
& \leq \sum_{t=1}^{T}\left(\nabla f_{t}\left(\mathbf{x}_{t}\right)^{\top}\left(\mathbf{x}_{t^{\prime}}-\mathbf{x}^{*}\right)-\frac{\beta}{2}\left\|\mathbf{x}_{t}-\mathbf{x}^{*}\right\|^{2}\right)+\sum_{t=1}^{T} L\left\|\mathbf{x}_{t}-\mathbf{x}_{t^{\prime}}\right\|
\end{aligned}
$$

where the first inequality is due to Assumption 3, and the last inequality is due to

$$
\begin{aligned}
\nabla f_{t}\left(\mathbf{x}_{t}\right)^{\top}\left(\mathbf{x}_{t}-\mathbf{x}_{t^{\prime}}\right) & \leq\left\|\nabla f_{t}\left(\mathbf{x}_{t}\right)\right\|\left\|\mathbf{x}_{t}-\mathbf{x}_{t^{\prime}}\right\| \\
& \leq L\left\|\mathbf{x}_{t}-\mathbf{x}_{t^{\prime}}\right\|
\end{aligned}
$$

To upper bound the right side of (4), we introduce the following lemma.

Lemma 2 For any $\mathrm{x} \in \mathcal{X}$, Algorithm 1 ensures

$$
\sum_{t=1}^{T}\left(\nabla f_{t}\left(\mathbf{x}_{t}\right)^{\top}\left(\mathbf{x}_{t^{\prime}}-\mathbf{x}\right)-\frac{\beta}{2}\left\|\mathbf{x}_{t}-\mathbf{x}\right\|^{2}\right) \leq \sum_{t=1}^{T} \beta R\left\|\mathbf{x}_{t}-\mathbf{x}_{t^{\prime}}\right\|+\sum_{t=s}^{T+d-1} \frac{d\left|\mathcal{F}_{t}\right| L^{2}}{2 h_{t}}
$$


Substituting (5) into (4), we have

$$
R_{T} \leq(\beta R+L) \sum_{t=1}^{T}\left\|\mathbf{x}_{t}-\mathbf{x}_{t^{\prime}}\right\|+\sum_{t=s}^{T+d-1} \frac{d\left|\mathcal{F}_{t}\right| L^{2}}{2 h_{t}} .
$$

According to the definition of $\mathbf{x}_{t+1}^{\prime}$, for any $t \in[T+d-1]$, it holds that

$$
\sum_{k \in \mathcal{F}_{t}} \nabla f_{k}\left(\mathbf{x}_{k}\right)=h_{t}\left(\mathbf{x}_{t}-\mathbf{x}_{t+1}^{\prime}\right)
$$

Moreover, since $\mathbf{x}_{t+1}=\Pi_{\mathcal{X}}\left(\mathbf{x}_{t+1}^{\prime}\right)$, for any $\mathbf{x} \in \mathcal{X}$, we have

$$
\left\|\mathbf{x}_{t+1}-\mathbf{x}\right\| \leq\left\|\mathbf{x}_{t+1}^{\prime}-\mathbf{x}\right\| .
$$

Then, it is not hard to verify that

$$
\begin{aligned}
\left\|\mathbf{x}_{t^{\prime}}-\mathbf{x}_{t}\right\| & \leq \sum_{i=t}^{t^{\prime}-1}\left\|\mathbf{x}_{i+1}-\mathbf{x}_{i}\right\| \\
& \leq \sum_{i=t}^{t^{\prime}-1}\left\|\mathbf{x}_{i+1}^{\prime}-\mathbf{x}_{i}\right\| \\
& =\sum_{i=\max (t, s)} \frac{\left\|\sum_{k \in \mathcal{F}_{i}} \nabla f_{k}\left(\mathbf{x}_{k}\right)\right\|}{h_{i}} \\
& \leq \sum_{i=\max (t, s)}^{t^{\prime}-1} \frac{\left|\mathcal{F}_{i}\right| L}{h_{i}}
\end{aligned}
$$

where the second inequality is due to (8), the equality is due to (7), and the last inequality is due to

$$
\left\|\sum_{k \in \mathcal{F}_{i}} \nabla f_{k}\left(\mathbf{x}_{k}\right)\right\| \leq \sum_{k \in \mathcal{F}_{i}}\left\|\nabla f_{k}\left(\mathbf{x}_{k}\right)\right\| \leq\left|\mathcal{F}_{i}\right| L .
$$

Substituting (9) into (6), we have

$$
R_{T} \leq\left(\beta R L+L^{2}\right) \sum_{t=1}^{T} \sum_{i=\max (t, s)}^{t^{\prime}-1} \frac{\left|\mathcal{F}_{i}\right|}{h_{i}}+\sum_{t=s}^{T+d-1} \frac{d\left|\mathcal{F}_{t}\right| L^{2}}{2 h_{t}} .
$$

Furthermore, we introduce the following lemma.

Lemma 3 Algorithm 1 ensures

$$
\sum_{t=1}^{T} \sum_{i=\max (t, s)}^{t^{\prime}-1} \frac{\left|\mathcal{F}_{i}\right|}{h_{i}} \leq 2 d \sum_{t=s}^{T+d-1} \frac{\left|\mathcal{F}_{t}\right|}{h_{t}}
$$

and

$$
\sum_{t=s}^{T+d-1} \frac{\left|\mathcal{F}_{t}\right|}{2 h_{t}} \leq \frac{1}{\beta}\left(1+\ln \frac{T}{\left|\mathcal{F}_{s}\right|}\right)
$$


Applying Lemma 3, we have

$$
\begin{aligned}
R_{T} & \leq\left(\beta R L+L^{2}\right) 2 d \sum_{t=s}^{T+d-1} \frac{\left|\mathcal{F}_{t}\right|}{h_{t}}+\sum_{t=s}^{T+d-1} \frac{d\left|\mathcal{F}_{t}\right| L^{2}}{2 h_{t}} \\
& \leq\left(4 \beta R L+5 L^{2}\right) \frac{d}{\beta}\left(1+\ln \frac{T}{\left|\mathcal{F}_{s}\right|}\right) .
\end{aligned}
$$

\subsection{Proof of Lemma 2}

First, we note that

$$
\begin{aligned}
& \sum_{t=1}^{T} \nabla f_{t}\left(\mathbf{x}_{t}\right)^{\top}\left(\mathbf{x}_{t^{\prime}}-\mathbf{x}\right) \\
= & \sum_{t=1}^{T+d-1} \sum_{k \in \mathcal{F}_{t}} \nabla f_{k}\left(\mathbf{x}_{k}\right)^{\top}\left(\mathbf{x}_{k+d_{k}-1}-\mathbf{x}\right) \\
= & \sum_{t=s}^{T+d-1} \sum_{k \in \mathcal{F}_{t}} \nabla f_{k}\left(\mathbf{x}_{k}\right)^{\top}\left(\mathbf{x}_{k+d_{k}-1}-\mathbf{x}\right) \\
= & \sum_{t=s}^{T+d-1} \sum_{k \in \mathcal{F}_{t}} \nabla f_{k}\left(\mathbf{x}_{k}\right)^{\top}\left(\mathbf{x}_{t}-\mathbf{x}\right) .
\end{aligned}
$$

where the last equality is due to $k+d_{k}-1=t$ for any $k \in \mathcal{F}_{t}$.

Substituting (7) into (11), we have

$$
\begin{aligned}
& \sum_{t=1}^{T} \nabla f_{t}\left(\mathbf{x}_{t}\right)^{\top}\left(\mathbf{x}_{t^{\prime}}-\mathbf{x}\right) \\
= & \sum_{t=s}^{T+d-1} h_{t}\left(\mathbf{x}_{t}-\mathbf{x}_{t+1}^{\prime}\right)^{\top}\left(\mathbf{x}_{t}-\mathbf{x}\right) \\
= & \sum_{t=s}^{T+d-1} \frac{h_{t}}{2}\left(\left\|\mathbf{x}_{t}-\mathbf{x}\right\|^{2}-\left\|\mathbf{x}_{t+1}^{\prime}-\mathbf{x}\right\|^{2}+\left\|\mathbf{x}_{t}-\mathbf{x}_{t+1}^{\prime}\right\|^{2}\right) \\
= & \sum_{t=s}^{T+d-1} \frac{h_{t}}{2}\left(\left\|\mathbf{x}_{t}-\mathbf{x}\right\|^{2}-\left\|\mathbf{x}_{t+1}^{\prime}-\mathbf{x}\right\|^{2}\right)+\sum_{t=s}^{T+d-1} \frac{\left\|\sum_{k \in \mathcal{F}_{t}} \nabla f_{k}\left(\mathbf{x}_{k}\right)\right\|^{2}}{2 h_{t}} \\
\leq & \sum_{t=s}^{T+d-1}\left(\frac{h_{t}}{2}\left(\left\|\mathbf{x}_{t}-\mathbf{x}\right\|^{2}-\left\|\mathbf{x}_{t+1}^{\prime}-\mathbf{x}\right\|^{2}\right)+\frac{\left|\mathcal{F}_{t}\right|^{2} L^{2}}{2 h_{t}}\right) \\
\leq & \sum_{t=s}^{T+d-1}\left(\frac{h_{t}}{2}\left(\left\|\mathbf{x}_{t}-\mathbf{x}\right\|^{2}-\left\|\mathbf{x}_{t+1}-\mathbf{x}\right\|^{2}\right)+\frac{\left|\mathcal{F}_{t}\right|^{2} L^{2}}{2 h_{t}}\right) \\
= & \sum_{t=s+1}^{T+d-1}\left\|\mathbf{x}_{t}-\mathbf{x}\right\|^{2}\left(\frac{h_{t}}{2}-\frac{h_{t-1}}{2}\right)+\sum_{t=s}^{T+d-1} \frac{\left|\mathcal{F}_{t}\right|^{2} L^{2}}{2 h_{t}}+\frac{h_{s}}{2}\left\|\mathbf{x}_{s}-\mathbf{x}\right\|^{2}-\frac{h_{T+d-1}\left\|\mathbf{x}_{T+d}-\mathbf{x}\right\|^{2}}{2}
\end{aligned}
$$


where the first inequality is due to (10), and the last inequality is due to (8).

According to Algorithm 1 and the virtual update, it is easy to verify that

$$
h_{t}=\frac{\sum_{i=s}^{t}\left|\mathcal{F}_{i}\right| \beta}{2}
$$

for any $t \in[s, T+d-1]$.

Combining the above equality with 12 , we have

$$
\begin{aligned}
& \sum_{t=1}^{T} \nabla f_{t}\left(\mathbf{x}_{t}\right)^{\top}\left(\mathbf{x}_{t^{\prime}}-\mathbf{x}\right) \\
\leq & \sum_{t=s+1}^{T+d-1}\left\|\mathbf{x}_{t}-\mathbf{x}\right\|^{2}\left(\frac{h_{t}}{2}-\frac{h_{t-1}}{2}\right)+\sum_{t=s}^{T+d-1} \frac{\left|\mathcal{F}_{t}\right|^{2} L^{2}}{2 h_{t}}+\frac{h_{s}}{2}\left\|\mathbf{x}_{s}-\mathbf{x}\right\|^{2} \\
= & \sum_{t=s+1}^{T+d-1} \frac{\left|\mathcal{F}_{t}\right| \beta}{4}\left\|\mathbf{x}_{t}-\mathbf{x}\right\|^{2}+\sum_{t=s}^{T+d-1} \frac{\left|\mathcal{F}_{t}\right|^{2} L^{2}}{2 h_{t}}+\frac{\left|\mathcal{F}_{s}\right| \beta}{4}\left\|\mathbf{x}_{s}-\mathbf{x}\right\|^{2} \\
= & \sum_{t=s}^{T+d-1} \frac{\left|\mathcal{F}_{t}\right| \beta}{4}\left\|\mathbf{x}_{t}-\mathbf{x}\right\|^{2}+\sum_{t=s}^{T+d-1} \frac{\left|\mathcal{F}_{t}\right|^{2} L^{2}}{2 h_{t}} .
\end{aligned}
$$

Moreover, we have

$$
\begin{aligned}
& \sum_{t=1}^{T}\left(\nabla f_{t}\left(\mathbf{x}_{t}\right)^{T}\left(\mathbf{x}_{t^{\prime}}-\mathbf{x}\right)-\frac{\beta}{2}\left\|\mathbf{x}_{t}-\mathbf{x}\right\|^{2}\right) \\
\leq & \sum_{t=s}^{T+d-1} \frac{\left|\mathcal{F}_{t}\right| \beta}{4}\left\|\mathbf{x}_{t}-\mathbf{x}\right\|^{2}+\sum_{t=s}^{T+d-1} \frac{\left|\mathcal{F}_{t}\right|^{2} L^{2}}{2 h_{t}}-\sum_{t=1}^{T} \frac{\beta}{2}\left\|\mathbf{x}_{t}-\mathbf{x}\right\|^{2} \\
= & \sum_{t=s}^{T+d-1} \frac{\left|\mathcal{F}_{t}\right| \beta}{4}\left\|\mathbf{x}_{t}-\mathbf{x}\right\|^{2}+\sum_{t=s}^{T+d-1} \frac{\left|\mathcal{F}_{t}\right|^{2} L^{2}}{2 h_{t}}-\sum_{t=s}^{T+d-1} \sum_{k \in \mathcal{F}_{t}} \frac{\beta}{2}\left\|\mathbf{x}_{k}-\mathbf{x}\right\|^{2} \\
\leq & \sum_{t=s}^{T+d-1} \frac{\left|\mathcal{F}_{t}\right| \beta}{4}\left\|\mathbf{x}_{t}-\mathbf{x}\right\|^{2}+\sum_{t=s}^{T+d-1} \frac{\left|\mathcal{F}_{t}\right|^{2} L^{2}}{2 h_{t}}+\sum_{t=s}^{T+d-1} \sum_{k \in \mathcal{F}_{t}} \frac{\beta}{2}\left(-\frac{\left\|\mathbf{x}_{t}-\mathbf{x}\right\|^{2}}{2}+\left\|\mathbf{x}_{t}-\mathbf{x}_{k}\right\|^{2}\right) \\
= & \sum_{t=s}^{T+d-1} \sum_{k \in \mathcal{F}_{t}} \frac{\beta}{2}\left\|\mathbf{x}_{t}-\mathbf{x}_{k}\right\|^{2}+\sum_{t=s}^{T+d-1} \frac{\left|\mathcal{F}_{t}\right|^{2} L^{2}}{2 h_{t}} \\
\leq & \sum_{t=s}^{T+d-1} \sum_{k \in \mathcal{F}_{t}} \beta R\left\|\mathbf{x}_{t}-\mathbf{x}_{k}\right\|+\sum_{t=s}^{T+d-1} \frac{\left|\mathcal{F}_{t}\right|^{2} L^{2}}{2 h_{t}}
\end{aligned}
$$

where the second inequality is due to

$$
\left\|\mathbf{x}_{t}-\mathbf{x}\right\|^{2} \leq 2\left\|\mathbf{x}_{t}-\mathbf{x}_{k}\right\|^{2}+2\left\|\mathbf{x}_{k}-\mathbf{x}\right\|^{2}
$$

and the last inequality is due to Assumption 2 .

Since $1 \leq d_{k} \leq d$, for any $t \in[T+d-1]$ and $k \in \mathcal{F}_{t}$, it is easy to verify that

$$
t-d+1 \leq k=t-d_{k}+1 \leq t
$$


which implies that $\left|\mathcal{F}_{t}\right| \leq t-(t-d+1)+1=d$ and

$$
\begin{aligned}
& \sum_{t=1}^{T}\left(\nabla f_{t}\left(\mathbf{x}_{t}\right)^{\top}\left(\mathbf{x}_{t^{\prime}}-\mathbf{x}\right)-\frac{\beta}{2}\left\|\mathbf{x}_{t}-\mathbf{x}\right\|^{2}\right) \\
\leq & \sum_{t=s}^{T+d-1} \sum_{k \in \mathcal{F}_{t}} \beta R\left\|\mathbf{x}_{t}-\mathbf{x}_{k}\right\|+\sum_{t=s}^{T+d-1} \frac{d\left|\mathcal{F}_{t}\right| L^{2}}{2 h_{t}} \\
= & \sum_{t=s}^{T+d-1} \sum_{k \in \mathcal{F}_{t}} \beta R\left\|\mathbf{x}_{k+d_{k}-1}-\mathbf{x}_{k}\right\|+\sum_{t=s}^{T+d-1} \frac{d\left|\mathcal{F}_{t}\right| L^{2}}{2 h_{t}} \\
= & \sum_{t=1}^{T} \beta R\left\|\mathbf{x}_{t}-\mathbf{x}_{t^{\prime}}\right\|+\sum_{t=s}^{T+d-1} \frac{d\left|\mathcal{F}_{t}\right| L^{2}}{2 h_{t}}
\end{aligned}
$$

where the first equality is due to $k+d_{k}-1=t$ for any $k \in \mathcal{F}_{t}$.

\subsection{Proof of Lemma 3}

First, it is not hard to verify that

$$
\begin{aligned}
\sum_{t=1}^{T} \sum_{i=\max (t, s)}^{t^{\prime}-1} \frac{\left|\mathcal{F}_{i}\right|}{h_{i}} & =\sum_{t=1}^{s-1} \sum_{i=s}^{t^{\prime}-1} \frac{\left|\mathcal{F}_{i}\right|}{h_{i}}+\sum_{t=s}^{T} \sum_{i=t}^{t^{\prime}-1} \frac{\left|\mathcal{F}_{i}\right|}{h_{i}} \\
& \leq \sum_{t=1}^{s-1} \sum_{i=s}^{t^{\prime}} \frac{\left|\mathcal{F}_{i}\right|}{h_{i}}+\sum_{t=s}^{T} \sum_{i=t}^{t^{\prime}} \frac{\left|\mathcal{F}_{i}\right|}{h_{i}} \\
& \leq \sum_{t=1}^{s-1} \sum_{i=s}^{T+d-1} \frac{\left|\mathcal{F}_{i}\right|}{h_{i}}+\sum_{t=s}^{T} \sum_{i=t}^{t^{\prime}} \frac{\left|\mathcal{F}_{i}\right|}{h_{i}} \\
& \leq \sum_{t=1}^{s-1} \sum_{i=s}^{T+d-1} \frac{\left|\mathcal{F}_{i}\right|}{h_{i}}+\sum_{t=s}^{T} \sum_{i=t}^{t+d-1} \frac{\left|\mathcal{F}_{i}\right|}{h_{i}} \\
& =\sum_{t=1}^{s-1} \sum_{i=s}^{T+d-1} \frac{\left|\mathcal{F}_{i}\right|}{h_{i}}+\sum_{i=0}^{d-1} \sum_{t=s+i}^{T+i} \frac{\left|\mathcal{F}_{t}\right|}{h_{t}} \\
& \leq \sum_{t=1}^{s-1} \sum_{i=s}^{T+d-1} \frac{\left|\mathcal{F}_{i}\right|}{h_{i}}+\sum_{i=0}^{d-1} \sum_{t=s}^{T+d-1} \frac{\left|\mathcal{F}_{t}\right|}{h_{t}} \\
& =(s-1+d) \sum_{t=s}^{T+d-1} \frac{\left|\mathcal{F}_{t}\right|}{h_{t}}
\end{aligned}
$$

where the second inequality is due to $t^{\prime}=t+d_{t}-1 \leq T+d-1$, and the third inequality is due to $t^{\prime}=t+d_{t}-1 \leq t+d-1$.

Since $1+d_{1}-1=d_{1} \leq d$, we note that $s \leq d$. Therefore, we have $s-1+d \leq 2 d$ and

$$
\sum_{t=1}^{T} \sum_{i=\max (t, s)}^{t^{\prime}-1} \frac{\left|\mathcal{F}_{i}\right|}{h_{i}} \leq 2 d \sum_{t=s}^{T+d-1} \frac{\left|\mathcal{F}_{t}\right|}{h_{t}} .
$$


Then, we continue to prove the second inequality in Lemma 3 with the following lemma.

Lemma 4 Let $a_{1}>0$ and $a_{2}, \cdots, a_{m} \geq 0$ be real numbers and let $f:(0,+\infty) \mapsto[0,+\infty)$ be a nonincreasing function. Then

$$
\sum_{i=1}^{m} a_{i} f\left(a_{1}+\cdots+a_{i}\right) \leq a_{1} f\left(a_{1}\right)+\int_{a_{1}}^{a_{1}+\cdots+a_{m}} f(x) d x .
$$

Let $f(x)=\frac{1}{x}$ and $a_{i}=\left|\mathcal{F}_{s+i-1}\right|$ for any $i \in[T+d-s]$. Then, we have

$$
a_{1}+\cdots+a_{T+d-s}=\sum_{t=s}^{T+d-1}\left|\mathcal{F}_{t}\right|=T .
$$

Because of $h_{t}=\frac{\sum_{i=s}^{t}\left|\mathcal{F}_{i}\right| \beta}{2}$ for any $t \in[s, T+d-1]$, we have

$$
\begin{aligned}
\sum_{t=s}^{T+d-1} \frac{\left|\mathcal{F}_{t}\right|}{2 h_{t}} & =\frac{1}{\beta} \sum_{i=1}^{T+d-s} a_{i} f\left(a_{1}+\cdots+a_{i}\right) \\
& \leq \frac{1}{\beta}\left(1+\int_{\left|\mathcal{F}_{s}\right|}^{T} \frac{1}{x} d x\right) \\
& =\frac{1}{\beta}\left(1+\ln \frac{T}{\left|\mathcal{F}_{s}\right|}\right)
\end{aligned}
$$

where the first inequality is due to Lemma 4 .

\subsection{Proof of Lemma 4}

Lemma 4 is inspired by Lemma 14 in Gaillard et al. (2014), which provides the following bound

$$
\sum_{i=2}^{m} a_{i} f\left(a_{1}+\cdots+a_{i-1}\right) \leq f\left(a_{1}\right)+\int_{a_{1}}^{a_{1}+\cdots+a_{m}} f(x) d x
$$

for $a_{2}, \cdots, a_{m} \in[0,1]$. It is not hard to prove Lemma 4 by slightly modifying the proof of Lemma 14 in Gaillard et al. (2014) to deal with $\sum_{i=1}^{m} a_{i} f\left(a_{1}+\cdots+a_{i}\right)$, instead of $\sum_{i=2}^{m} a_{i} f\left(a_{1}+\cdots+a_{i-1}\right)$. We include the proof for completeness.

Let $s_{i}=a_{1}+\cdots+a_{i}$ for any $i \in[m]$. Then, for any $i=2, \cdots, m$, we have

$$
a_{i} f\left(s_{i}\right)=\int_{s_{i-1}}^{s_{i}} f\left(s_{i}\right) d x \leq \int_{s_{i-1}}^{s_{i}} f(x) d x
$$

where the inequality is due to the fact that $f(x)$ is a nonincreasing function.

Then, we have

$$
\begin{aligned}
\sum_{i=1}^{m} a_{i} f\left(s_{i}\right) & =a_{1} f\left(a_{1}\right)+\sum_{i=2}^{m} a_{i} f\left(s_{i}\right) \\
& \leq a_{1} f\left(a_{1}\right)+\int_{s_{1}}^{s_{m}} f(x) d x .
\end{aligned}
$$




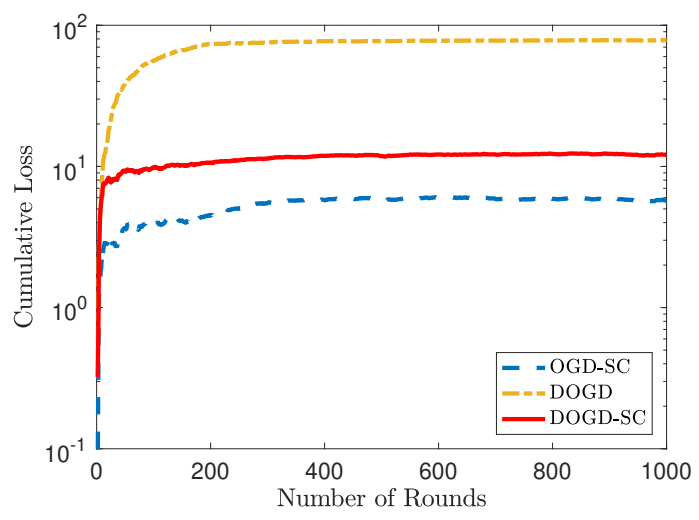

(a) Low Delayed Setting

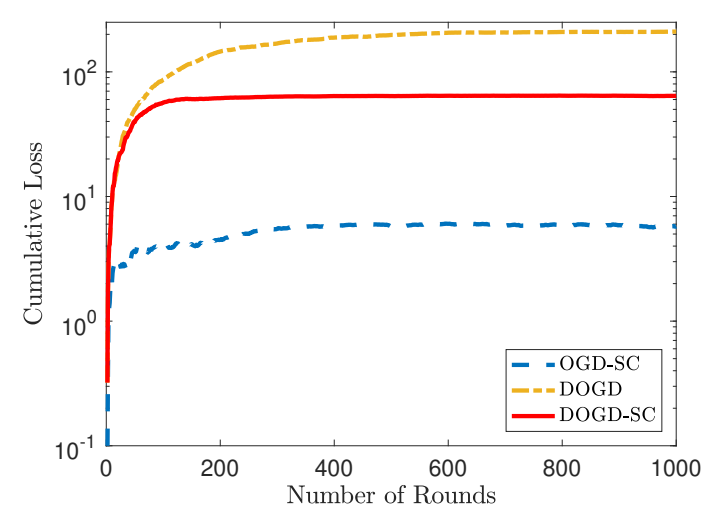

(b) High Delayed Setting

Figure 1: Comparisons of our Algorithm 1 against OGD-SC and DOGD.

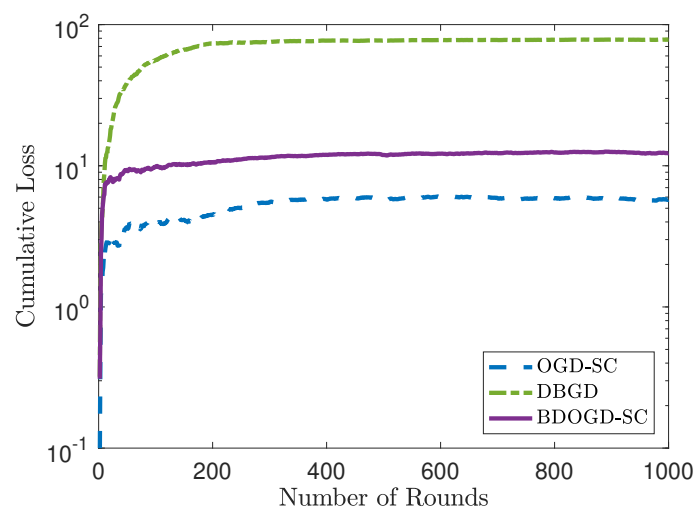

(a) Low Delayed Setting

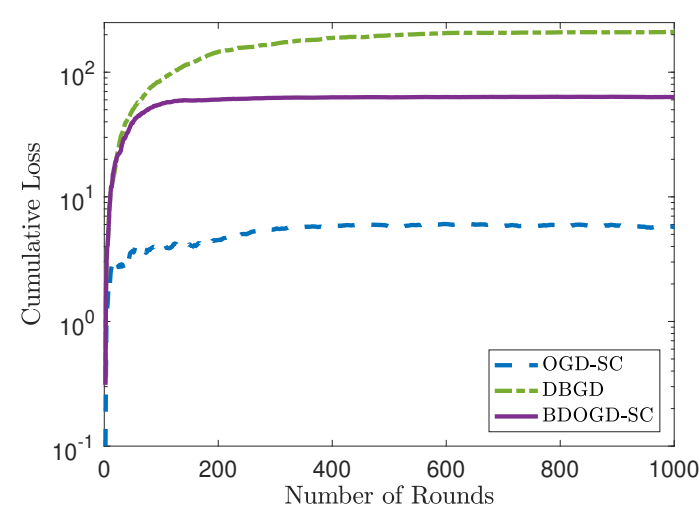

(b) High Delayed Setting

Figure 2: Comparisons of our Algorithm 2 against OGD-SC and DBGD.

\section{Experiments}

In this section, we provide numerical experiments to verify the performance of our DOGD-SC and BDOGD-SC for strongly convex functions.

The experimental setup is inspired by Li et al. (2019). In each round $t$, the player chooses a decision $\mathbf{x}_{t}$ from the unit ball $\mathcal{X}=\left\{\mathbf{x} \in \mathbb{R}^{10} \mid\|\mathbf{x}\| \leq 1\right\}$. Then, the loss function is generated as $f_{t}(\mathbf{x})=\|\mathbf{x}\|^{2}+\mathbf{b}_{t}^{\top} \mathbf{x}$, where each element of $\mathbf{b}_{t}$ is uniformly sampled from $[-1,1]$. In this problem, the decision set $\mathcal{X}$ satisfies Assumption 2 with $R=1$ and Assumption 4 with $r=1$. Each function $f_{t}(\mathbf{x})$ is 2-strongly convex and 2-smooth, which satisfies Assumptions 3 and 5 , respectively. Moreover, since $\nabla f_{t}(\mathbf{x})=2 \mathbf{x}+\mathbf{b}_{t}$, we have

$$
\left\|\nabla f_{t}(\mathbf{x})\right\| \leq 2\|\mathbf{x}\|+\left\|\mathbf{b}_{t}\right\| \leq 2+\sqrt{10}
$$

for any $\mathbf{x} \in \mathcal{X}$, which implies that each function $f_{t}(\mathbf{x})$ satisfies Assumption 1 with $L=2+\sqrt{10}$.

We set $T=1000$, and consider two cases: the low delayed setting, in which the delays are periodically generated with length $2,3,2,1,4,1,3$, and the high delayed setting, in which 
the delays are periodically generated with length $20,30,20,10,40,10,30$. In the low delayed setting, the maximum delay is $d=4=O(1)$. In the other setting, the maximum delay $d=40$ is on the order of $O(\sqrt{T})$.

We compared DOGD-SC and BDOGD-SC against online gradient descent for strongly convex functions (OGD-SC) (Hazan et al., 2007), DOGD (Quanrud and Khashabi, 2015) and DBGD (Li et al., 2019). Specifically, OGD-SC is implemented without delay, and other algorithms are implemented with delayed feedback. The parameters of these algorithms are set as what their theoretical results suggest. For OGD-SC, we set the learning rate as $\eta_{t}=1 /(\beta t)$, where $\beta=2$ in our experiments. For DOGD and DBGD, a constant learning rate $\eta=1 /(L \sqrt{T+D})$ is used. Moreover, we set $\delta=\ln T / T$ for BDOGD-SC and set $\delta=1 /(T+D)$ for DBGD. Furthermore, we initialize the decision as $\mathbf{x}_{1}=1 / \sqrt{10}$ for algorithms in the full information setting, and $\mathbf{x}_{1}=(1-\delta) \mathbf{1} / \sqrt{10}$ for algorithms in the bandit setting, where 1 denotes the vector with each entry equal 1 .

Fig. 1 shows the cumulative loss for OGD-SC, DOGD and our DOGD-SC. We find that in both low and high delayed settings, our DOGD-SC is better than DOGD. Moreover, in the low delayed setting, the performance of our DOGD-SC is significantly better than DOGD, and close to OGD-SC. These results confirm that our DOGD-SC can utilize the strong convexity to achieve better regret. In our experiments, we also find that the performance of our BDOGD-SC is almost the same as that of DOGD-SC, and better than DBGD which is very close to DOGD. To make a clear presentation, we put the results of BDOGD-SC and DBGD in Fig. 2, which confirms the theoretical guarantee of BDOGD-SC. Since BDOGD-SC and DBGD query the function at 11 points per round, the average results are reported.

\section{Conclusion}

In this paper, we consider the problem of $\mathrm{OCO}$ with unknown delays, and present a variant of DOGD for strongly convex functions called DOGD-SC. According to our analysis, it enjoys a better regret bound of $O(d \log T)$ for strongly convex functions. Furthermore, we propose a bandit variant of DOGD-SC to handle the bandit setting, and achieve the same regret bound. Experimental results verify the performance of DOGD-SC and its bandit variant for strongly convex functions.

\section{References}

Jacob D. Abernethy, Peter L. Bartlett, Alexander Rakhlin, and Ambuj Tewari. Optimal stragies and minimax lower bounds for online convex games. In Proceedings of the 21st Annual Conference on Learning Theory, pages 415-424, 2008.

Alekh Agarwal, Ofer Dekel, and Lin Xiao. Optimal algorithms for online convex optimization with multi-point bandit feedback. In Proceedings of the 23rd Annual Conference on Learning Theory, pages 28-40, 2010.

Amit Agarwal, Elad Hazan, Satyen Kale, and Robert E. Schapire. Algorithms for portfolio management based on the Newton method. In Proceedings of the 23rd International Conference on Machine Learning, pages 9-16, 2006. 
Avrim Blum and Adam Kalai. Universal portfolios with and without transaction costs. Machine Learning, 35(3):193-205, 1999.

Nicolò Cesa-Bianchi and Gabor Lugosi. Prediction, Learning, and Games. Cambridge University Press, 2006.

John Duchi, Elad Hazan, and Yoram Singer. Adaptive subgradient methods for online learning and stochastic optimization. Journal of Machine Learning Research, 12:2121-2159, 2011.

Abraham D. Flaxman, Adam Tauman Kalai, and H. Brendan McMahan. Online convex optimization in the bandit setting: Gradient descent without a gradient. In Proceedings of the 16th Annual ACM-SIAM Symposium on Discrete Algorithms, pages 385-394, 2005.

Pierre Gaillard, Gilles Stoltz, and Tim van Erven. A second-order bound with excess losses. In Proceedings of the 27th Annual Conference on Learning Theory, pages 176-196, 2014.

Elad Hazan. Introduction to online convex optimization. Foundations and Trends in Optimization, 2(3-4):157-325, 2016.

Elad Hazan, Amit Agarwal, and Satyen Kale. Logarithmic regret algorithms for online convex optimization. Machine Learning, 69(2):169-192, 2007.

Xinran He, Junfeng Pan, Ou Jin, Tianbing Xu, Bo Liu, Tao Xu, Yanxin Shi, Antoine Atallah, Ralf Herbrich, Stuart Bowers, and Joaquin Q. Candela. Practical lessons from predicting clicks on ads at facebook. In Proceedings of the 8th International Workshop on Data Mining for Online Advertising, pages 1-9, 2014.

Amélie Héliou, Panayotis Mertikopoulos, and Zhengyuan Zhou. Gradient-free online learning in games with delayed rewards. In Proceedings of the 37 th International Conference on Machine Learning, pages 4172-4181, 2020.

Pooria Joulani, András György, and Csaba Szepesvári. Online learning under delayed feedback. In Proceedings of the 30th International Conference on Machine Learning, pages 1453-1461, 2013.

Pooria Joulani, András György, and Csaba Szepesvári. Delay-tolerant online convex optimization: Unified analysis and adaptive-gradient algorithms. Proceedings of the 30th AAAI Conference on Artificial Intelligence, pages 1744-1750, 2016.

Yuchin Juan, Damien Lefortier, and Olivier Chapelle. Field-aware factorization machines in a real-world online advertising system. In Proceedings of the 26th International Conference on World Wide Web Companion, pages 680-688, 2017.

Daniel Khashabi, Kent Quanrud, and Amirhossein Taghvaei. Adversarial delays in online strongly-convex optimization. arXiv:1605.06201v1, 2016.

John Langford, Alexander J. Smola, and Martin Zinkevich. Slow learners are fast. In Advances in Neural Information Processing Systems 22, pages 2331-2339, 2009. 
Bingcong Li, Tianyi Chen, and Georgios B. Giannakis. Bandit online learning with unknown delays. In Proceedings of the 22nd International Conference on Artificial Intelligence and Statistics, pages 993-1002, 2019.

Haipeng Luo, Chen-Yu Wei, and Kai Zheng. Efficient online portfolio with logarithmic regret. In Advances in Neural Information Processing Systems 31, pages 8235-8245, 2018.

H. Brendan McMahan and Matthew Streeter. Adaptive bound optimization for online convex optimization. In Proceedings of the 23rd Conference on Learning Theory, pages 244-256, 2010.

H. Brendan McMahan and Matthew Streeter. Delay-tolerant algorithms for asynchronous distributed online learning. In Advances in Neural Information Processing Systems 27, pages 2915-2923, 2014.

H. Brendan McMahan, Gary Holt, D. Sculley, Michael Young, Dietmar Ebner, Julian Grady, Lan Nie, Todd Phillips, Eugene Davydov, Daniel Golovin, Sharat Chikkerur, Dan Liu, Martin Wattenberg, Arnar Mar Hrafnkelsson, Tom Boulos, and Jeremy Kubica. Ad click prediction: a view from the trenches. In Proceedings of the 19th ACM SIGKDD International Conference on Knowledge Discovery and Data Mining, pages 1222-1230, 2013.

Chris Mesterharm. On-line learning with delayed label feedback. In Proceedings of the 16th International Conference on Algorithmic Learning Theory, pages 399-413, 2005.

Kent Quanrud and Daniel Khashabi. Online learning with adversarial delays. In Advances in Neural Information Processing Systems 28, pages 1270-1278, 2015.

Ankan Saha and Ambuj Tewari. Improved regret guarantees for online smooth convex optimization with bandit feedback. In Proceedings of the 14 th International Conference on Artificial Intelligence and Statistics, pages 636-642, 2011.

Shai Shalev-Shwartz. Online learning and online convex optimization. Foundations and Trends in Machine Learning, 4(2):107-194, 2011.

Ohad Shamir and Liran Szlak. Online learning with local permutations and delayed feedback. In Proceedings of the 34th International Conference on Machine Learning, pages 3086-3094, 2017.

Marcelo J. Weinberger and Erik Ordentlich. On delayed prediction of individual sequences. IEEE Transactions on Information Theory, 48(7):1959-1976, 2002.

Martin Zinkevich. Online convex programming and generalized infinitesimal gradient ascent. In Proceedings of the 20th International Conference on Machine Learning, pages 928-936, 2003. 


\section{Appendix A. Proof of Theorem 2}

This proof is inspired by the work of Agarwal et al. (2010), which combined the $(n+1)$-point gradient estimator with OGD, and proved the average regret bound in the non-delayed setting. In this paper, we combine the $(n+1)$-point gradient estimator with DOGD-SC, and prove the average regret bound in the general delayed setting.

According to Assumption 11, for any $i=1, \cdots, n$, we have

$$
f_{t}\left(\mathbf{x}_{t}+\delta \mathbf{e}_{i}\right) \leq f_{t}\left(\mathbf{x}_{t}\right)+L\left\|\delta \mathbf{e}_{i}\right\| \leq f_{t}\left(\mathbf{x}_{t}\right)+L \delta
$$

which implies that

$$
\begin{aligned}
& \frac{1}{n+1} \sum_{t=1}^{T} \sum_{i=0}^{n} f_{t}\left(\mathbf{x}_{t}+\delta \mathbf{e}_{i}\right)-\sum_{t=1}^{T} f_{t}(\mathbf{x}) \\
\leq & \sum_{t=1}^{T} f_{t}\left(\mathbf{x}_{t}\right)+\sum_{t=1}^{T} \frac{n L \delta}{n+1}-\sum_{t=1}^{T} f_{t}(\mathbf{x}) \\
\leq & \sum_{t=1}^{T} f_{t}\left(\mathbf{x}_{t}\right)-\sum_{t=1}^{T}\left(f_{t}((1-\delta / r) \mathbf{x})-L \delta\|\mathbf{x}\| / r\right)+T L \delta \\
\leq & \sum_{t=1}^{T} f_{t}\left(\mathbf{x}_{t}\right)-\sum_{t=1}^{T} f_{t}((1-\delta / r) \mathbf{x})+\frac{T L R \delta}{r}+T L \delta .
\end{aligned}
$$

for any $\mathrm{x} \in \mathcal{X}$.

Then, we only need to upper bound $\sum_{t=1}^{T} f_{t}\left(\mathbf{x}_{t}\right)-\sum_{t=1}^{T} f_{t}((1-\delta / r) \mathbf{x})$. To this end, we start by defining

$$
\ell_{t}(\mathbf{x})=f_{t}(\mathbf{x})+\left(\tilde{\mathbf{g}}_{t}-\nabla f_{t}\left(\mathbf{x}_{t}\right)\right)^{\top} \mathbf{x} .
$$

It is easy to verify that $\ell_{t}(\mathbf{x})$ is also $\beta$-strongly convex, and $\nabla \ell_{t}\left(\mathbf{x}_{t}\right)=\tilde{\mathbf{g}}_{t}$. Therefore, Algorithm 2 is actually performing Algorithm 1 on the functions $\ell_{t}(\mathbf{x})$ over the decision set $\mathcal{X}_{\delta}$.

Moreover, under Assumptions 1 and 5 , Lemma 1 shows

$$
\left\|\tilde{\mathbf{g}}_{t}\right\| \leq \sqrt{n} L \text { and }\left\|\tilde{\mathbf{g}}_{t}-\nabla f_{t}\left(\mathbf{x}_{t}\right)\right\| \leq \frac{\sqrt{n} \alpha \delta}{2}
$$

which implies that

$$
\left\|\nabla \ell_{t}(\mathbf{x})\right\| \leq\left\|\nabla f_{t}(\mathbf{x})\right\|+\left\|\tilde{\mathbf{g}}_{t}-\nabla f_{t}\left(\mathbf{x}_{t}\right)\right\| \leq L+\frac{\sqrt{n} \alpha \delta}{2} .
$$

Define $\tilde{L}=L+\frac{\sqrt{n} \alpha \delta}{2}$. Applying Theorem 1 to the functions $\ell_{t}(\mathbf{x})$, for any $\mathbf{x} \in \mathcal{X}$, we have

$$
\begin{aligned}
& \sum_{t=1}^{T} \ell_{t}\left(\mathbf{x}_{t}\right)-\sum_{t=1}^{T} \ell_{t}((1-\delta / r) \mathbf{x}) \\
\leq & \sum_{t=1}^{T} \ell_{t}\left(\mathbf{x}_{t}\right)-\min _{\mathbf{x}^{\prime} \in \mathcal{X}_{\delta}} \sum_{t=1}^{T} \ell_{t}\left(\mathbf{x}^{\prime}\right) \\
\leq & \left(4 \beta R \tilde{L}+5 \tilde{L}^{2}\right) \frac{d}{\beta}\left(1+\ln \frac{T}{\left|\mathcal{F}_{s}\right|}\right) .
\end{aligned}
$$




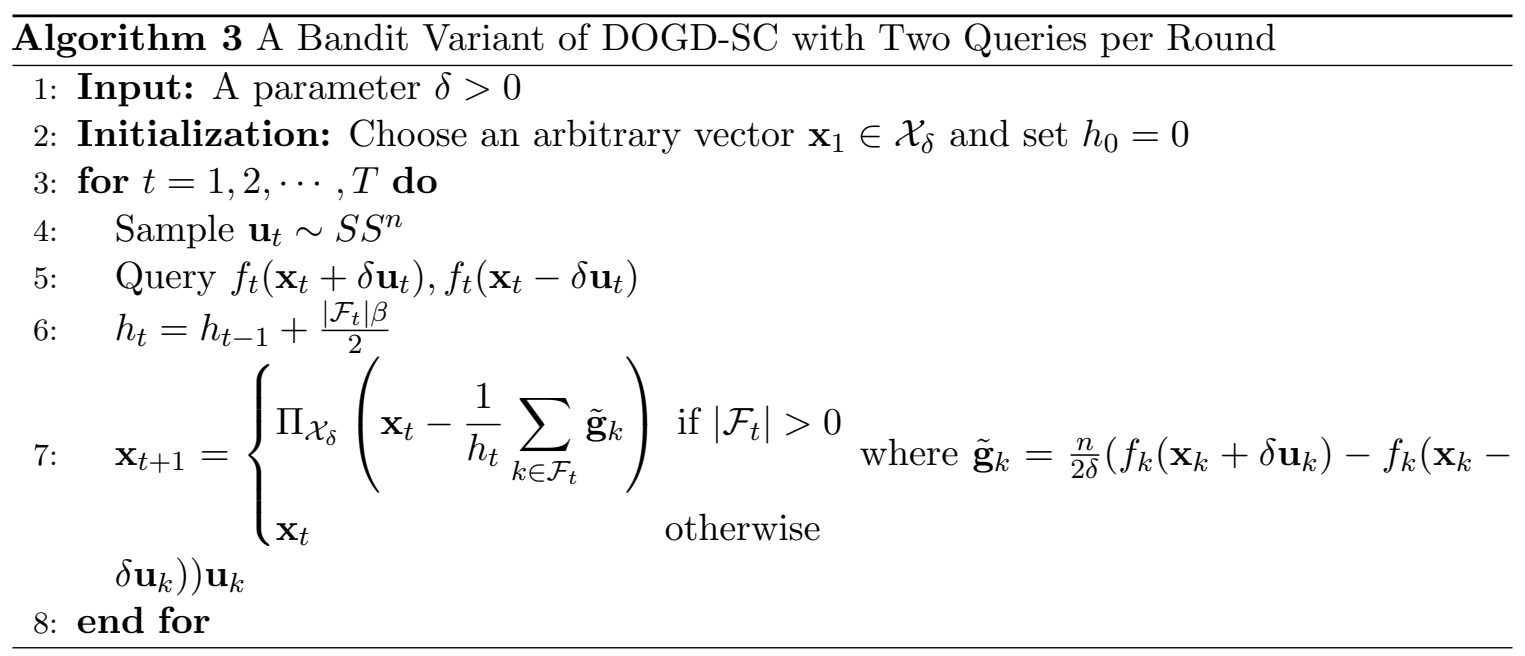

Furthermore, for any $\mathbf{x} \in \mathcal{X}$, we have

$$
\begin{aligned}
& \sum_{t=1}^{T} f_{t}\left(\mathbf{x}_{t}\right)-\sum_{t=1}^{T} f_{t}((1-\delta / r) \mathbf{x}) \\
= & \sum_{t=1}^{T} \ell_{t}\left(\mathbf{x}_{t}\right)-\sum_{t=1}^{T} \ell_{t}((1-\delta / r) \mathbf{x})+\sum_{t=1}^{T}\left(\tilde{\mathbf{g}}_{t}-\nabla f_{t}\left(\mathbf{x}_{t}\right)\right)^{\top}\left(\mathbf{x}_{t}-(1-\delta / r) \mathbf{x}\right) \\
\leq & \sum_{t=1}^{T} \ell_{t}\left(\mathbf{x}_{t}\right)-\sum_{t=1}^{T} \ell_{t}((1-\delta / r) \mathbf{x})+\sum_{t=1}^{T}\left\|\tilde{\mathbf{g}}_{t}-\nabla f_{t}\left(\mathbf{x}_{t}\right)\right\|\left\|\mathbf{x}_{t}-(1-\delta / r) \mathbf{x}\right\| \\
\leq & \left(4 \beta R \tilde{L}+5 \tilde{L}^{2}\right) \frac{d}{\beta}\left(1+\ln \frac{T}{\left|\mathcal{F}_{s}\right|}\right)+\sum_{t=1}^{T} \sqrt{n} \alpha \delta R .
\end{aligned}
$$

Combining with (14), for any $\mathrm{x} \in \mathcal{X}$, we have

$$
\begin{aligned}
& \frac{1}{n+1} \sum_{t=1}^{T} \sum_{i=0}^{n} f_{t}\left(\mathbf{x}_{t}+\delta \mathbf{e}_{i}\right)-\sum_{t=1}^{T} f_{t}(\mathbf{x}) \\
\leq & \left(4 \beta R \tilde{L}+5 \tilde{L}^{2}\right) \frac{d}{\beta}\left(1+\ln \frac{T}{\left|\mathcal{F}_{s}\right|}\right)+\sum_{t=1}^{T} \sqrt{n} \alpha \delta R+\frac{T L R \delta}{r}+T L \delta .
\end{aligned}
$$

We complete this proof by substituting $\delta=\frac{c \ln T}{T}$ into the above inequality.

\section{Appendix B. A Bandit Variant of DOGD-SC with Two Queries per Round}

In Section 3.2, we have proposed BDOGD-SC for the bandit setting, which requires $n+1$ queries per round. To reduce the number of queries, we further present a bandit variant of DOGD-SC with only two queries per round, for the case where the time stamp of each delayed feedback is known. 
According to Agarwal et al. (2010), for a function $f(\mathbf{x}): \mathcal{X} \mapsto \mathbb{R}$ and a point $\mathbf{x} \in \mathcal{X}_{\delta}$, the two-point gradient estimator queries

$$
f(\mathbf{x}+\delta \mathbf{u}), f(\mathbf{x}-\delta \mathbf{u})
$$

where $\mathbf{u}$ is uniformly at random sampled from the unit sphere $S S^{n}$, and estimates the gradient $\nabla f(\mathbf{x})$ by

$$
\tilde{\mathbf{g}}=\frac{n}{2 \delta}(f(\mathbf{x}+\delta \mathbf{u})-f(\mathbf{x}-\delta \mathbf{u})) \mathbf{u}
$$

Combining DOGD-SC with this technique, a new bandit variant of DOGD-SC is outlined in Algorithm 3, and named as a bandit variant of DOGD-SC with two queries per round. Specifically, given $0<\delta<r$ and $\mathcal{X}_{\delta}=(1-\delta / r) \mathcal{X}$, in each round $t \in[T]$, the learner queries $f_{t}\left(\mathbf{x}_{t}+\delta \mathbf{u}_{t}\right), f_{t}\left(\mathbf{x}_{t}-\delta \mathbf{u}_{t}\right)$, where $\mathbf{x}_{t} \in \mathcal{X}_{\delta}$ and $\mathbf{u}_{t}$ is uniformly at random sampled from the unit sphere $S S^{n}$. After receiving the feedback

$$
\left\{f_{k}\left(\mathbf{x}_{k}+\delta \mathbf{u}_{k}\right), f_{k}\left(\mathbf{x}_{k}-\delta \mathbf{u}_{k}\right) \mid k+d_{k}-1=t\right\}
$$

we can compute the approximate gradient

$$
\tilde{\mathbf{g}}_{k}=\frac{n}{2 \delta}\left(f_{k}\left(\mathbf{x}_{k}+\delta \mathbf{u}_{k}\right)-f_{k}\left(\mathbf{x}_{k}-\delta \mathbf{u}_{k}\right)\right) \mathbf{u}_{k} .
$$

for any $k \in \mathcal{F}_{t}$ according to 15 , which needs to use the time stamp $k$ to match the feedback $\left\{f_{k}\left(\mathbf{x}_{k}+\delta \mathbf{u}_{k}\right), f_{k}\left(\mathbf{x}_{k}-\delta \mathbf{u}_{k}\right)\right\}$ with the random vector $\mathbf{u}_{k}$. Then, we compute the sum $\sum_{k \in \mathcal{F}_{t}} \tilde{\mathbf{g}}_{k}$, and update $\mathbf{x}_{t}$ as

$$
\mathbf{x}_{t+1}= \begin{cases}\Pi_{\mathcal{X}_{\delta}}\left(\mathbf{x}_{t}-\frac{1}{h_{t}} \sum_{k \in \mathcal{F}_{t}} \tilde{\mathbf{g}}_{k}\right) & \text { if }\left|\mathcal{F}_{t}\right|>0 \\ \mathbf{x}_{t} & \text { otherwise }\end{cases}
$$

Following previous studies for the bandit setting (Flaxman et al., 2005; Saha and Tewari, 2011), we assume that the adversary is oblivious, and establish the following theorem.

Theorem 3 Let $\mathbf{x}$ be an arbitrary vector in the set $\mathcal{X}$. Let $\mathbf{x}_{t, 1}=\mathbf{x}_{t}+\delta \mathbf{u}_{t}$ and $\mathbf{x}_{t, 2}=\mathbf{x}_{t}-\delta \mathbf{u}_{t}$. Define $\tilde{L}=2 L+$ Ln. Let $\delta=\frac{c \ln T}{T}$, where $c>0$ is a constant such that $\delta<r$. Under Assumptions 1, 2, 3 and 4, Algorithm 2 ensures

$$
\mathbb{E}\left[\frac{1}{2} \sum_{t=1}^{T} \sum_{i=1}^{2} f_{t}\left(\mathbf{x}_{t, i}\right)-\sum_{t=1}^{T} f_{t}(\mathbf{x})\right] \leq\left(4 \beta R \tilde{L}+5 \tilde{L}^{2}\right) \frac{d}{\beta}\left(1+\ln \frac{T}{\left|\mathcal{F}_{s}\right|}\right)+3 c L \ln T+\frac{R L \ln T}{r} .
$$

where $s=\min \left\{t|t \in[T+d-1],| \mathcal{F}_{t} \mid>0\right\}$.

\section{Appendix C. Proof of Theorem 3}

This proof is inspired by the work of Agarwal et al. (2010), which analyzed the expected regret for the combination of the two-point gradient estimator and OGD in the non-delayed setting. 
We first introduce the $\delta$-smoothed version of a function $f(\mathbf{x})$ and the corresponding properties, which will be used in the following proof. For a function $f(\mathbf{x})$, its $\delta$-smoothed version is defined as

$$
\hat{f}(\mathbf{x})=\mathbb{E}_{\mathbf{u} \sim \mathcal{B}^{n}}[f(\mathbf{x}+\delta \mathbf{u})]
$$

and satisfies the following two lemmas.

Lemma 5 (Lemma 1 in Flaxman et al. (2005)) Let $\delta>0$, we have

$$
\nabla \hat{f}(\mathbf{x})=\mathbb{E}_{\mathbf{u} \sim S S^{n}}\left[\frac{n}{\delta} f(\mathbf{x}+\delta \mathbf{u}) \mathbf{u}\right]
$$

where $S S^{n}$ denotes the unit sphere in $\mathbb{R}^{n}$.

Lemma 6 (Derived from Lemma 2.6 of Hazan (2016)) Let $f(\mathbf{x}): \mathbb{R}^{n} \rightarrow \mathbb{R}$ be $\beta$-strongly convex and L-Lipschitz over a convex and compact set $\mathcal{X} \subset \mathbb{R}^{n}$. Then, $\hat{f}(\mathbf{x})$ has the following properties.

- $\hat{f}(\mathbf{x})$ is $\beta$-strongly convex over $\mathcal{X}_{\delta}$;

- $|\hat{f}(\mathbf{x})-f(\mathbf{x})| \leq \delta L$ for any $\mathbf{x} \in \mathcal{X}_{\delta}$;

- $\hat{f}(\mathbf{x})$ is L-Lipschitz over $\mathcal{X}_{\delta}$.

Let $\hat{\mathbf{x}}=(1-\delta / r) \mathbf{x}$. We have

$$
\begin{aligned}
& \frac{1}{2} \sum_{t=1}^{T} \sum_{i=1}^{2} f_{t}\left(\mathbf{x}_{t, i}\right)-\sum_{t=1}^{T} f_{t}(\mathbf{x}) \\
= & \frac{1}{2} \sum_{t=1}^{T}\left(f_{t}\left(\mathbf{x}_{t}+\delta \mathbf{u}_{t}\right)+f_{t}\left(\mathbf{x}_{t}-\delta \mathbf{u}_{t}\right)\right)-\sum_{t=1}^{T} f_{t}(\mathbf{x}) \\
\leq & \frac{1}{2} \sum_{t=1}^{T}\left(f_{t}\left(\mathbf{x}_{t}\right)+L\left\|\delta \mathbf{u}_{t}\right\|+f_{t}\left(\mathbf{x}_{t}\right)+L\left\|\delta \mathbf{u}_{t}\right\|\right)-\sum_{t=1}^{T}\left(f_{t}(\hat{\mathbf{x}})-L \delta\|\mathbf{x}\| / r\right) \\
\leq & \sum_{t=1}^{T} f_{t}\left(\mathbf{x}_{t}\right)-\sum_{t=1}^{T} f_{t}(\hat{\mathbf{x}})+L T \delta+\frac{R L T \delta}{r} \\
\leq & \sum_{t=1}^{T}\left(\hat{f}_{t}\left(\mathbf{x}_{t}\right)+\delta L\right)-\sum_{t=1}^{T}\left(\hat{f}_{t}(\hat{\mathbf{x}})-\delta L\right)+L T \delta+\frac{R L T \delta}{r} \\
= & \sum_{t=1}^{T} \hat{f}_{t}\left(\mathbf{x}_{t}\right)-\sum_{t=1}^{T} \hat{f}_{t}(\hat{\mathbf{x}})+3 L T \delta+\frac{R L T \delta}{r}
\end{aligned}
$$

where the first inequality is due to Assumption 1 and the last inequality is due to Lemma 6 ,

Then, we only need to upper bound $\sum_{t=1}^{T} \hat{f}_{t}\left(\mathbf{x}_{t}\right)-\sum_{t=1}^{T} \hat{f}_{t}(\hat{\mathbf{x}})$. Similar to the proof of Theorem 2, we define

$$
\ell_{t}(\mathbf{x})=\hat{f}_{t}(\mathbf{x})+\left(\tilde{\mathbf{g}}_{t}-\nabla \hat{f}_{t}\left(\mathbf{x}_{t}\right)\right)^{\top} \mathbf{x} .
$$


According to Lemma 5, we have

$$
\begin{aligned}
\mathbb{E}_{\mathbf{u}_{t}}\left[\tilde{\mathbf{g}}_{t}\right] & =\mathbb{E}_{\mathbf{u}_{t}}\left[\frac{n}{2 \delta}\left(f_{t}\left(\mathbf{x}_{t}+\delta \mathbf{u}_{t}\right)-f_{t}\left(\mathbf{x}_{t}-\delta \mathbf{u}_{t}\right)\right) \mathbf{u}_{t}\right] \\
& =\mathbb{E}_{\mathbf{u}_{t}}\left[\frac{n}{\delta} f_{t}\left(\mathbf{x}_{t}+\delta \mathbf{u}_{t}\right) \mathbf{u}_{t}\right]=\nabla \hat{f}_{t}\left(\mathbf{x}_{t}\right)
\end{aligned}
$$

where the second equality is due to the fact that the distribution of $\mathbf{u}_{t}$ is symmetric.

Then, we have $\mathbb{E}_{\mathbf{u}_{t}}\left[\tilde{\mathbf{g}}_{t}-\nabla \hat{f}_{t}\left(\mathbf{x}_{t}\right)\right]=0$, which implies that

$$
\mathbb{E}\left[\sum_{t=1}^{T}\left(\hat{f}_{t}\left(\mathbf{x}_{t}\right)-\hat{f}_{t}(\hat{\mathbf{x}})\right)\right]=\mathbb{E}\left[\sum_{t=1}^{T}\left(\ell_{t}\left(\mathbf{x}_{t}\right)-\ell_{t}(\hat{\mathbf{x}})\right)\right]
$$

Therefore, we only need to derive an upper bound of $\sum_{t=1}^{T} \ell_{t}\left(\mathbf{x}_{t}\right)-\sum_{t=1}^{T} \ell_{t}(\hat{\mathbf{x}})$.

According to the definition of $\ell_{t}(\mathbf{x})$, it is easy to verify that $\nabla \ell_{t}\left(\mathbf{x}_{t}\right)=\tilde{\mathbf{g}}_{t}$. Moreover, from Lemma $6, \hat{f}_{t}(\mathbf{x})$ is $\beta$-strongly convex, which implies that $\ell_{t}(\mathbf{x})$ is also $\beta$-strongly convex. Therefore, Algorithm 3 is actually performing Algorithm 1 on the functions $\ell_{t}(\mathbf{x})$ over the decision set $\mathcal{X}_{\delta}$.

Before using Theorem 1, we need to prove that $\ell_{t}(\mathbf{x})$ is also Lipschitz. From Lemma 6 , $\hat{f}_{t}(\mathbf{x})$ is $L$-Lipschitz. So, for any $\mathbf{x}, \mathbf{y} \in \mathcal{X}_{\delta}$, it is not hard to verify that

$$
\begin{aligned}
\left|\ell_{t}(\mathbf{x})-\ell_{t}(\mathbf{y})\right| & \leq\left|\hat{f}_{t}(\mathbf{x})-\hat{f}_{t}(\mathbf{y})\right|+\left|\left(\tilde{\mathbf{g}}_{t}-\nabla \hat{f}_{t}\left(\mathbf{x}_{t}\right)\right)^{\top}(\mathbf{x}-\mathbf{y})\right| \\
& \leq L\|\mathbf{x}-\mathbf{y}\|+\left\|\tilde{\mathbf{g}}_{t}-\nabla \hat{f}_{t}\left(\mathbf{x}_{t}\right)\right\|\|\mathbf{x}-\mathbf{y}\| \\
& \leq\left(L+\left\|\tilde{\mathbf{g}}_{t}\right\|+\left\|\nabla \hat{f}_{t}\left(\mathbf{x}_{t}\right)\right\|\right)\|\mathbf{x}-\mathbf{y}\| \\
& \leq(2 L+L n)\|\mathbf{x}-\mathbf{y}\|
\end{aligned}
$$

where the last inequality is due to $\left\|\nabla \hat{f}_{t}\left(\mathbf{x}_{t}\right)\right\| \leq L$ and

$$
\begin{aligned}
\left\|\tilde{\mathbf{g}}_{t}\right\| & =\frac{n}{2 \delta}\left\|\left(f_{t}\left(\mathbf{x}_{t}+\delta \mathbf{u}_{t}\right)-f_{t}\left(\mathbf{x}_{t}-\delta \mathbf{u}_{t}\right)\right) \mathbf{u}_{t}\right\| \\
& =\frac{n}{2 \delta}\left|f_{t}\left(\mathbf{x}_{t}+\delta \mathbf{u}_{t}\right)-f_{t}\left(\mathbf{x}_{t}-\delta \mathbf{u}_{t}\right)\right| \\
& \leq \frac{n}{2 \delta} L\left\|2 \delta \mathbf{u}_{t}\right\|=n L .
\end{aligned}
$$

Let $\tilde{L}=2 L+L n$. Since $\ell_{t}(\mathbf{x})$ is $\beta$-strongly convex and $\tilde{L}$-Lipschitz. Applying Theorem 1 to the functions $\ell_{t}(\mathbf{x})$, we have

$$
\sum_{t=1}^{T}\left(\ell_{t}\left(\mathbf{x}_{t}\right)-\ell_{t}(\hat{\mathbf{x}})\right) \leq\left(4 \beta R \tilde{L}+5 \tilde{L}^{2}\right) \frac{d}{\beta}\left(1+\ln \frac{T}{\left|\mathcal{F}_{s}\right|}\right)
$$


Combining (16), (17) and (18), we have

$$
\begin{aligned}
& \mathbb{E}\left[\frac{1}{2} \sum_{t=1}^{T} \sum_{i=1}^{2} f_{t}\left(\mathbf{x}_{t, i}\right)-\sum_{t=1}^{T} f_{t}(\mathbf{x})\right] \\
\leq & \mathbb{E}\left[\sum_{t=1}^{T}\left(\hat{f}_{t}\left(\mathbf{x}_{t}\right)-\hat{f}_{t}(\hat{\mathbf{x}})\right)\right]+3 L T \delta+\frac{R L T \delta}{r} \\
= & \mathbb{E}\left[\sum_{t=1}^{T}\left(\ell_{t}\left(\mathbf{x}_{t}\right)-\ell_{t}(\hat{\mathbf{x}})\right)\right]+3 L T \delta+\frac{R L T \delta}{r} \\
\leq & \left(4 \beta R \tilde{L}+5 \tilde{L}^{2}\right) \frac{d}{\beta}\left(1+\ln \frac{T}{\left|\mathcal{F}_{s}\right|}\right)+3 L T \delta+\frac{R L T \delta}{r} .
\end{aligned}
$$

We complete this proof by substituting $\delta=\frac{c \ln T}{T}$ into the above inequality.

\section{Appendix D. Proof of Lemma 6}

The first and second properties have been presented in Lemma 2.6 of Hazan (2016). The last property is proved by

$$
\begin{aligned}
|\hat{f}(\mathbf{x})-\hat{f}(\mathbf{y})| & =\left|\mathbb{E}_{\mathbf{u} \sim \mathcal{B}^{d}}[f(\mathbf{x}+\delta \mathbf{u})]-\mathbb{E}_{\mathbf{u} \sim \mathcal{B}^{d}}[f(\mathbf{y}+\delta \mathbf{u})]\right| \\
& =\left|\mathbb{E}_{\mathbf{u} \sim \mathcal{B}^{d}}[f(\mathbf{x}+\delta \mathbf{u})-f(\mathbf{y}+\delta \mathbf{u})]\right| \\
& \leq \mathbb{E}_{\mathbf{u} \sim \mathcal{B}^{d}}[|f(\mathbf{x}+\delta \mathbf{u})-f(\mathbf{y}+\delta \mathbf{u})|] \\
& \leq \mathbb{E}_{\mathbf{u} \sim \mathcal{B}^{d}}[L\|\mathbf{x}-\mathbf{y}\|] \\
& =L\|\mathbf{x}-\mathbf{y}\|
\end{aligned}
$$

where the first inequality is due to Jensen's inequality, and the second inequality is due to the fact that $f(\mathbf{x})$ is $L$-Lipschitz over $\mathcal{X}$. 